\title{
A New Selective Haloform-Type Reaction Yielding 3-Hydroxy-2,2-Difluoroacids. A Theoretical Study of the Mechanism
}

Santiago Olivella, Albert Solé, Óscar Jiménez, Mª Pilar Bosch, and Angel Guerrero

\author{
SUPPORTING INFORMATION
}

List of Compounds
$\stackrel{\mathrm{OH}}{\mathrm{RR}^{\prime} \mathrm{CCF}_{2} \mathrm{COCF}_{3}}$

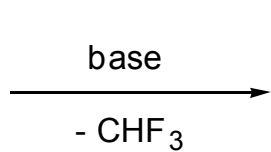
$\stackrel{\mathrm{OH}}{\mathrm{RR}^{\prime} \mathrm{CCF}_{2} \mathrm{CO}_{2} \mathrm{H}}$
1a: $\mathrm{R}=\mathrm{C}_{6} \mathrm{H}_{13}, \mathrm{R}^{\prime}=\mathrm{H}$
2a: $\mathrm{R}=\mathrm{C}_{6} \mathrm{H}_{13}, \mathrm{R}^{\prime}=\mathrm{H}$
1b: $\mathrm{R}=\mathrm{C}_{8} \mathrm{H}_{17}, \mathrm{R}^{\prime}=\mathrm{H}$
2b: $\mathrm{R}=\mathrm{C}_{8} \mathrm{H}_{17}, \mathrm{R}^{\prime}=\mathrm{H}$
1c: $\mathrm{R}=\mathrm{Z10}-\mathrm{C}_{15} \mathrm{H}_{29}, \mathrm{R}^{\prime}=\mathrm{H}$
2c: $\mathrm{R}=\mathrm{Z10}-\mathrm{C}_{15} \mathrm{H}_{29}, \mathrm{R}^{\prime}=\mathrm{H}$
1d: $\mathrm{R}=\mathrm{Ph}, \mathrm{R}^{\prime}=\mathrm{H}$
2d: $\mathrm{R}=\mathrm{Ph}, \mathrm{R}^{\prime}=\mathrm{H}$
1e: $\mathrm{R}=\mathrm{PhCH}_{2} \mathrm{CH}_{2}, \mathrm{R}^{\prime}=\mathrm{H}$
2e: $\mathrm{R}=\mathrm{PhCH}_{2} \mathrm{CH}_{2}, \mathrm{R}^{\prime}=\mathrm{H}$
1f: $\mathrm{R}=\mathrm{PhCH}=\mathrm{CH}, \mathrm{R}^{\prime}=\mathrm{H}$
2f: $\mathrm{R}=\mathrm{PhCH}=\mathrm{CH}, \mathrm{R}^{\prime}=\mathrm{H}$
1g: $\mathrm{R}=\mathrm{p}-\mathrm{CF}_{3} \mathrm{COCF}_{2} \mathrm{CHOHPh}, \mathrm{R}^{\prime}=\mathrm{H}$
2g: R=p- $\mathrm{CF}_{3} \mathrm{COCF}_{2} \mathrm{CHOHPh}, \mathrm{R}^{\prime}=\mathrm{H}$
1h: $\mathrm{R}=\mathrm{p}-\mathrm{CH}_{3} \mathrm{OPh}, \mathrm{R}^{\prime}=\mathrm{H}$
2h: $\mathrm{R}=\mathrm{p}-\mathrm{CH}_{3} \mathrm{OPh}, \mathrm{R}^{\prime}=\mathrm{H}$
1i: $\mathrm{R}=\mathrm{p}-\mathrm{O}_{2} \mathrm{NPh}, \mathrm{R}^{\prime}=\mathrm{H}$
2i: $\mathrm{R}=\mathrm{p}-\mathrm{O}_{2} \mathrm{NPh}, \mathrm{R}^{\prime}=\mathrm{H}$
1j: $\mathrm{R}=\mathrm{C}_{4} \mathrm{H}_{9}, \mathrm{R}^{\prime}=\mathrm{H}$
2j: $\mathrm{R}=\mathrm{C}_{4} \mathrm{H}_{9}, \mathrm{R}^{\prime}=\mathrm{H}$
1k: $\mathrm{R}=\mathrm{CH}_{3} \mathrm{COCH}_{2} \mathrm{CH}_{2}, \mathrm{R}^{\prime}=\mathrm{H}$
2k: $\mathrm{R}=\mathrm{CH}_{3} \mathrm{COCH}_{2} \mathrm{CH}_{2}, \mathrm{R}^{\prime}=\mathrm{H}$

Table S1. Base promoted conversion of 4-alkyl-4-hydroxy-3,3-difluoromethyl trifluoromethyl ketones $\mathbf{1}$ into 3-hydroxy-2,2-difluoroacids $\mathbf{2}^{1}$

\begin{tabular}{|c|c|c|c|c|c|}
\hline Substrate & Base $^{2}$ & Solvent & Time (h) & Product & Yield $^{3}(\%)$ \\
\hline $1 \mathrm{a}$ & $\mathrm{NaH}$ & THF & 4 & $2 a$ & 70 \\
\hline $1 \mathbf{a}$ & $\mathrm{NaOH}(1 \mathrm{~N})$ & $\mathrm{THF} / \mathrm{H}_{2} \mathrm{O}$ & 27 & $2 a$ & $80^{4}$ \\
\hline $1 \mathbf{a}$ & $\mathrm{NaOH}$ & $\mathrm{THF} / \mathrm{H}_{2} \mathrm{O}$ & 17 & $2 a$ & 89 \\
\hline $1 \mathrm{~b}$ & $\mathrm{NaOH}$ & $\mathrm{THF} / \mathrm{H}_{2} \mathrm{O}$ & 23 & $2 \mathrm{~b}$ & 83 \\
\hline $1 b$ & $\mathrm{NaH}$ & THF & 3 & $2 \mathrm{~b}$ & 87 \\
\hline 1c & $\mathrm{NaOH}$ & $\mathrm{THF} / \mathrm{H}_{2} \mathrm{O}$ & 16 & $2 c$ & 92 \\
\hline 1d & $\mathrm{NaOH}$ & $\mathrm{THF} / \mathrm{H}_{2} \mathrm{O}$ & 22 & 2d & 69 \\
\hline $1 \mathrm{e}$ & $\mathrm{NaOH}$ & $\mathrm{THF} / \mathrm{H}_{2} \mathrm{O}$ & 17 & $2 e$ & 89 \\
\hline 1f & $\mathrm{NaOH}$ & $\mathrm{THF} / \mathrm{H}_{2} \mathrm{O}$ & 15.5 & $2 f$ & 79 \\
\hline
\end{tabular}




\begin{tabular}{|c|c|c|c|c|c|}
\hline $\mathbf{1 g}$ & $\mathrm{NaOH}$ & $\mathrm{THF} / \mathrm{H}_{2} \mathrm{O}$ & 22 & $\mathbf{2 g}$ & 90 \\
\hline $\mathbf{1 h}$ & $\mathrm{NaOH}$ & $\mathrm{THF} / \mathrm{H}_{2} \mathrm{O}$ & 17 & $\mathbf{2 h}$ & 86 \\
\hline $\mathbf{1 i}$ & $\mathrm{NaOH}$ & $\mathrm{THF} / \mathrm{H}_{2} \mathrm{O}$ & 21 & $\mathbf{2 i}$ & 84 \\
\hline $\mathbf{1 j}$ & $\mathrm{NaOH}$ & $\mathrm{THF} / \mathrm{H}_{2} \mathrm{O}$ & 22 & $\mathbf{2 j}$ & 81 \\
\hline $\mathbf{1 k}$ & $\mathrm{NaOH}$ & $\mathrm{THF} / \mathrm{H}_{2} \mathrm{O}$ & 21 & $\mathbf{2 k}$ & 84 \\
\hline
\end{tabular}

${ }^{1}$ All reactions were conducted at room temperature.

${ }^{2} \mathrm{NaH}$ (5 equivalents) and $5 \mathrm{~N} \mathrm{NaOH}$ aq. soln. (20 equivalents) were used as base, except for substrate 1a in entry 2.

${ }^{3}$ Isolated yield.

${ }^{4}$ Yield based on ${ }^{19} \mathrm{~F}$ NMR.

Cartesian coordinates of the B3LYP/6-311+G(d,p)-optimized geometries:

$\mathbf{R}$

$\begin{array}{lrrr}\text { C } & 0.000000 & 0.000000 & 0.000000 \\ \text { C } & 0.000000 & 0.000000 & 1.528208 \\ \text { C } & 1.420248 & 0.000000 & 2.122776 \\ \text { O } & -0.779994 & -1.037270 & 2.087361 \\ \text { H } & 0.417271 & -0.927083 & -0.401310 \\ \text { H } & -1.030670 & 0.086228 & -0.347097 \\ \text { H } & 0.571002 & 0.841595 & -0.397071 \\ \text { H } & -0.442180 & 0.930872 & 1.895375 \\ \text { H } & -0.442960 & -1.883295 & 1.767158 \\ \text { F } & 2.099167 & 1.102920 & 1.671334 \\ \text { F } & 1.361262 & 0.095761 & 3.485193 \\ \text { C } & 2.272861 & -1.259107 & 1.818212 \\ \text { O } & 1.778129 & -2.307983 & 1.518637 \\ \text { C } & 3.828931 & -1.131947 & 1.905462 \\ \text { F } & 4.288882 & -0.580715 & 0.765815 \\ \text { F } & 4.209277 & -0.365662 & 2.935383 \\ \text { F } & 4.381867 & -2.337244 & 2.042844\end{array}$

$\mathrm{HO}^{-}$

$\begin{array}{llll}\mathrm{H} & 0.000000 & 0.000000 & 0.000000 \\ \mathrm{O} & 0.000000 & 0.000000 & 0.966075\end{array}$

INT

$\begin{array}{lrrr}\mathrm{C} & 0.000000 & 0.000000 & 0.000000 \\ \mathrm{C} & 0.000000 & 0.000000 & 1.531644 \\ \mathrm{C} & 1.406417 & 0.000000 & 2.178818 \\ \mathrm{O} & -0.773485 & -1.061815 & 2.051637 \\ \mathrm{H} & 0.386447 & -0.943297 & -0.385068 \\ \mathrm{H} & -1.034689 & 0.115322 & -0.335668 \\ \mathrm{H} & 0.597896 & 0.820235 & -0.405711 \\ \mathrm{H} & -0.454107 & 0.937243 & 1.879819 \\ \mathrm{H} & -0.130136 & -1.824898 & 2.040124 \\ \mathrm{~F} & 2.114981 & 1.083866 & 1.664146 \\ \mathrm{~F} & 1.218648 & 0.303511 & 3.508603\end{array}$




$\begin{array}{lllr}\text { C } & 2.232697 & -1.369785 & 2.079048 \\ \text { O } & 1.430643 & -2.395845 & 1.911768 \\ \text { C } & 3.339755 & -1.303481 & 0.950302 \\ \text { F } & 2.828846 & -1.006687 & -0.276656 \\ \text { F } & 4.332164 & -0.404041 & 1.170493 \\ \text { F } & 3.944091 & -2.505915 & 0.821630 \\ \text { O } & 3.052287 & -1.473864 & 3.277991 \\ \text { H } & 2.661511 & -2.248482 & 3.699661\end{array}$

\section{TS1}

$\begin{array}{lrrr}\mathrm{C} & 0.000000 & 0.000000 & 0.000000 \\ \mathrm{C} & 0.000000 & 0.000000 & 1.527600 \\ \mathrm{C} & 1.414267 & 0.000000 & 2.138005 \\ \mathrm{O} & -0.784949 & -1.055203 & 2.065166 \\ \mathrm{H} & 0.453147 & -0.910154 & -0.392703 \\ \mathrm{H} & -1.036890 & 0.067975 & -0.340646 \\ \mathrm{H} & 0.564211 & 0.848039 & -0.393395 \\ \mathrm{H} & -0.453689 & 0.927800 & 1.893529 \\ \mathrm{H} & -0.243142 & -1.858784 & 1.980960 \\ \mathrm{~F} & 2.106505 & 1.077532 & 1.666449 \\ \mathrm{~F} & 1.289416 & 0.217228 & 3.510324 \\ \mathrm{C} & 2.214643 & -1.316574 & 2.003383 \\ \mathrm{O} & 1.624101 & -2.380102 & 1.954929 \\ \mathrm{C} & 3.119256 & -1.316218 & -0.501835 \\ \mathrm{~F} & 2.551961 & -2.207861 & -1.422399 \\ \mathrm{~F} & 3.769035 & -0.407390 & -1.342964 \\ \mathrm{~F} & 4.277687 & -2.119474 & -0.035258 \\ \mathrm{O} & 3.511880 & -1.162901 & 2.305908 \\ \mathrm{H} & 4.013085 & -1.772937 & 1.727653\end{array}$

\section{CX1}

$\begin{array}{lrrr}\text { C } & -2.640746 & 1.399358 & -1.086231 \\ \text { C } & -2.617039 & 1.400704 & 0.444947 \\ \text { C } & -1.177268 & 1.387915 & 0.990041 \\ \text { O } & -3.383272 & 0.343788 & 0.992936 \\ \text { H } & -2.193779 & 0.484677 & -1.483799 \\ \text { H } & -3.680126 & 1.443157 & -1.418290 \\ \text { H } & -2.101461 & 2.257381 & -1.494021 \\ \text { H } & -3.059503 & 2.330331 & 0.818488 \\ \text { H } & -2.826186 & -0.453151 & 0.933085 \\ \text { F } & -0.536028 & 2.514777 & 0.542963 \\ \text { F } & -1.211308 & 1.492505 & 2.366674 \\ \text { C } & -0.382088 & 0.098697 & 0.644576 \\ \text { O } & -0.980632 & -0.966368 & 0.702536 \\ \text { C } & 2.740788 & -1.637742 & -1.851181 \\ \text { F } & 2.590961 & -2.997906 & -2.005917 \\ \text { F } & 4.097610 & -1.528056 & -1.624787 \\ \text { F } & 2.264000 & -1.573130 & -0.222748\end{array}$




$\begin{array}{rrrr}\mathrm{O} & 0.850301 & 0.310080 & 0.335429 \\ \mathrm{H} & 1.408381 & -0.552371 & 0.075355\end{array}$

\section{TS2}

$\begin{array}{lrrr}\text { C } & -2.514464 & 1.404000 & -1.142795 \\ \text { C } & -2.558102 & 1.348758 & 0.386634 \\ \text { C } & -1.143091 & 1.350434 & 0.994201 \\ \text { O } & -3.322348 & 0.256594 & 0.863313 \\ \text { H } & -2.035799 & 0.511966 & -1.554889 \\ \text { H } & -3.538395 & 1.446363 & -1.519494 \\ \text { H } & -1.970808 & 2.284078 & -1.493824 \\ \text { H } & -3.037993 & 2.253152 & 0.775190 \\ \text { H } & -2.755371 & -0.531115 & 0.797850 \\ \text { F } & -0.516542 & 2.514572 & 0.633490 \\ \text { F } & -1.235103 & 1.377517 & 2.369851 \\ \text { C } & -0.306244 & 0.102961 & 0.612505 \\ \text { O } & -0.847803 & -0.985807 & 0.645790 \\ \text { C } & 2.395188 & -1.618169 & -1.523141 \\ \text { F } & 2.321979 & -3.002146 & -1.720822 \\ \text { F } & 3.666054 & -1.331075 & -2.049711 \\ \text { F } & 2.725672 & -1.590126 & -0.043823 \\ \text { O } & 0.920548 & 0.380387 & 0.285308 \\ \text { H } & 1.436934 & -0.420412 & -0.025399\end{array}$

\section{CX2}

$\begin{array}{lrrr}\text { C } & -1.960614 & 2.140066 & -0.844748 \\ \text { C } & -2.211846 & 1.385731 & 0.465892 \\ \text { C } & -1.152852 & 0.295568 & 0.699296 \\ \text { O } & -3.515613 & 0.835967 & 0.509821 \\ \text { H } & -2.019573 & 1.456777 & -1.695989 \\ \text { H } & -2.734699 & 2.902280 & -0.963699 \\ \text { H } & -0.980232 & 2.623046 & -0.848131 \\ \text { H } & -2.123569 & 2.081526 & 1.309484 \\ \text { H } & -3.424619 & -0.040088 & 0.064491 \\ \text { F } & 0.089950 & 0.908936 & 0.720464 \\ \text { F } & -1.318872 & -0.216352 & 1.979108 \\ \text { C } & -1.182238 & -0.902771 & -0.319576 \\ \text { O } & -2.346956 & -1.280042 & -0.599949 \\ \text { C } & 2.840846 & -0.605726 & -0.460392 \\ \text { F } & 3.358545 & -0.635428 & 0.791430 \\ \text { F } & 3.511946 & -1.536728 & -1.185966 \\ \text { F } & 3.151032 & 0.603069 & -0.990132 \\ \text { O } & -0.088865 & -1.333779 & -0.719641 \\ \text { H } & 1.760968 & -0.789392 & -0.464986\end{array}$

\section{$\mathrm{CH}_{3} \mathrm{CHOHCF}_{2} \mathrm{CO}_{2} \mathrm{H}$}
C $\quad 0.000000$
0.000000
0.000000
C $\quad 0.000000$
0.000000
1.529174 


$\begin{array}{lrrr}\mathrm{C} & 1.419999 & 0.000000 & 2.122284 \\ \mathrm{O} & -0.776761 & -1.043409 & 2.082354 \\ \mathrm{H} & 0.433723 & -0.921090 & -0.397190 \\ \mathrm{H} & -1.032751 & 0.066237 & -0.345722 \\ \mathrm{H} & 0.555573 & 0.851388 & -0.398142 \\ \mathrm{H} & -0.447632 & 0.928388 & 1.895338 \\ \mathrm{H} & -0.378425 & -1.883445 & 1.817271 \\ \mathrm{~F} & 2.113706 & 1.075680 & 1.636204 \\ \mathrm{~F} & 1.358934 & 0.151468 & 3.482092 \\ \mathrm{C} & 2.235141 & -1.289056 & 1.863840 \\ \mathrm{O} & 1.727327 & -2.335664 & 1.544640 \\ \mathrm{O} & 3.540457 & -1.107919 & 2.060857 \\ \mathrm{H} & 3.985726 & -1.959540 & 1.925534 \\ & & & \\ \mathrm{CF}_{3}{ }^{-} & & & \\ \mathrm{C} & 0.000000 & 0.000000 & 0.554545 \\ \mathrm{~F} & 0.000000 & 1.272209 & -0.123232 \\ \mathrm{~F} & 1.101765 & -0.636104 & -0.123232 \\ \mathrm{~F} & -1.101765 & -0.636104 & -0.123232\end{array}$

\section{$\mathrm{CH}_{3} \mathrm{CHOHCF}_{2} \mathrm{CO}_{2}^{-}$}

$\begin{array}{lrrr}\mathrm{C} & 0.000000 & 0.000000 & 0.000000 \\ \mathrm{C} & 0.000000 & 0.000000 & 1.533712 \\ \mathrm{C} & 1.433434 & 0.000000 & 2.093837 \\ \mathrm{O} & -0.743478 & -1.086764 & 2.051874 \\ \mathrm{H} & 0.494970 & -0.897825 & -0.379459 \\ \mathrm{H} & 1.035450 & -0.004860 & -0.350599 \\ \mathrm{H} & 0.510246 & 0.879226 & -0.401937 \\ \mathrm{H} & -0.469982 & 0.923297 & 1.896927 \\ \mathrm{H} & -0.078559 & -1.820693 & 2.108204 \\ \mathrm{~F} & 2.076140 & 1.122497 & 1.602548 \\ \mathrm{~F} & 1.363278 & 0.206995 & 3.469569 \\ \mathrm{C} & 2.260871 & -1.317092 & 1.830060 \\ \mathrm{O} & 1.561782 & -2.358089 & 1.997069 \\ \mathrm{O} & 3.443849 & -1.193687 & 1.506460\end{array}$

$\mathrm{CHF}_{3}$

$\begin{array}{lrrr}\text { H } & 0.000000 & 0.000000 & 1.431094 \\ \text { C } & 0.000000 & 0.000000 & 0.341331 \\ \text { F } & 0.000000 & 1.258461 & -0.128855 \\ \text { F } & 1.089859 & -0.629231 & -0.128855 \\ \text { F } & -1.089859 & -0.629231 & -.0128855\end{array}$

TS3

$\begin{array}{rrrr}\mathrm{C} & 0.000000 & 0.000000 & 0.000000 \\ \mathrm{C} & 0.000000 & 0.000000 & 1.529108 \\ \mathrm{C} & 1.420702 & 0.000000 & 2.079394 \\ \mathrm{O} & -0.674107 & -1.169897 & 2.023470\end{array}$




$\begin{array}{lrrr}\text { H } & 0.628130 & -0.813169 & -0.377473 \\ \text { H } & -1.020050 & -0.149664 & -0.365694 \\ \text { H } & 0.387130 & 0.945867 & -0.388256 \\ \text { H } & -0.570264 & 0.864772 & 1.900098 \\ \text { H } & 0.038864 & -1.779411 & 2.263879 \\ \text { F } & 2.050439 & 1.141701 & 1.524435 \\ \text { F } & 1.217756 & 0.487567 & 3.520784 \\ \text { C } & 3.056415 & -2.218709 & 3.650611 \\ \text { O } & 3.054955 & -3.394289 & 3.392510 \\ \text { C } & 4.270512 & -1.371350 & 3.163263 \\ \text { F } & 4.541152 & -1.558474 & 1.867782 \\ \text { F } & 4.174755 & -0.058786 & 3.403955 \\ \text { F } & 5.370219 & -1.806396 & 3.859768 \\ \text { O } & 2.238265 & -1.637876 & 4.514074 \\ \text { H } & 1.972401 & -0.719151 & 4.223648\end{array}$

\section{CX3}

$\begin{array}{lrrr}\mathrm{C} & 0.000000 & 0.000000 & 0.000000 \\ \mathrm{C} & 0.000000 & 0.000000 & 1.528863 \\ \mathrm{C} & 1.423972 & 0.000000 & 2.075515 \\ \mathrm{O} & -0.647554 & -1.186444 & 2.021470 \\ \mathrm{H} & 0.642945 & -0.801321 & -0.378361 \\ \mathrm{H} & -1.016858 & -0.169046 & -0.366217 \\ \mathrm{H} & 0.368423 & 0.953746 & -0.387673 \\ \mathrm{H} & -0.578779 & 0.857258 & 1.902271 \\ \mathrm{H} & 0.090933 & -1.739509 & 2.320838 \\ \mathrm{~F} & 2.051615 & 1.142471 & 1.525508 \\ \mathrm{~F} & 1.170084 & 0.547984 & 3.535016 \\ \mathrm{C} & 2.925267 & 0.046677 & 6.318831 \\ \mathrm{O} & 3.525050 & -0.432934 & 7.249094 \\ \mathrm{C} & 2.641799 & 1.584885 & 6.316360 \\ \mathrm{~F} & 3.236209 & 2.193132 & 5.272337 \\ \mathrm{~F} & 1.319596 & 1.841867 & 6.253452 \\ \mathrm{~F} & 3.107905 & 2.163385 & 7.437406 \\ \mathrm{O} & 2.481079 & -0.617922 & 5.289852 \\ \mathrm{H} & 1.977792 & -0.103028 & 4.561347\end{array}$

\section{TS4}

$\begin{array}{lrrr}\text { C } & -1.916002 & -0.774564 & -3.832174 \\ \text { C } & -2.075800 & -0.616070 & -2.320412 \\ \text { C } & -0.746424 & -0.299248 & -1.645787 \\ \text { O } & -2.574992 & -1.838985 & -1.746642 \\ \text { H } & -1.115409 & -1.487508 & -4.054610 \\ \text { H } & -2.847854 & -1.153329 & -4.262627 \\ \text { H } & -1.666536 & 0.182625 & -4.298017 \\ \text { H } & -2.826681 & 0.158768 & -2.103752 \\ \text { F } & -1.786595 & -2.243969 & -1.353317 \\ \text { F } & -0.235722 & 0.849767 & -2.315437\end{array}$




$\begin{array}{lrrr}\text { F } & -1.174945 & 0.329344 & -0.330703 \\ \text { C } & 1.204167 & -0.637404 & 1.954103 \\ \text { O } & 1.765698 & -1.178849 & 2.872373 \\ \text { C } & 1.384174 & 0.901967 & 1.756333 \\ \text { F } & 1.617038 & 1.228669 & 0.478421 \\ \text { F } & 0.283694 & 1.563767 & 2.172157 \\ \text { F } & 2.420960 & 1.354002 & 2.487393 \\ \text { O } & 0.427130 & -1.249791 & 1.097703 \\ \text { H } & 0.000465 & -0.693619 & 0.385977\end{array}$

\section{CX4}

$\begin{array}{llll}\text { C } & -2.768512 & 0.697573 & 0.643021\end{array}$

$\begin{array}{llll}\text { C } & -1.996527 & 1.893557 & 0.076085\end{array}$

$\begin{array}{llll}\text { C } & -1.514894 & 1.618814 & -1.347513\end{array}$

$\begin{array}{llll}\text { O } & -0.902782 & 2.262222 & 0.884405\end{array}$

$\begin{array}{llll}\mathrm{H} & -2.125773 & -0.185412 & 0.670857\end{array}$

$\begin{array}{llll}\mathrm{H} & -3.072184 & 0.933150 & 1.665848\end{array}$

$\begin{array}{llll}\mathrm{H} & -3.659733 & 0.476516 & 0.049745\end{array}$

$\begin{array}{llll}\mathrm{H} & -2.655187 & 2.768861 & 0.019301\end{array}$

$\begin{array}{llll}\mathrm{H} & -0.287174 & 1.488979 & 0.924690\end{array}$

$\begin{array}{llll}\text { F } & -2.579832 & 1.223319 & 2.151702\end{array}$

$\begin{array}{llll}\text { F } & -1.028914 & 2.772179 & -1.924091\end{array}$

$\begin{array}{llll}\mathrm{C} & 1.392677 & -0.667259 & 1.087717\end{array}$

$\begin{array}{llll}\text { O } & 2.034016 & -0.420027 & 2.106974\end{array}$

$\begin{array}{llll}\text { C } & 1.766234 & -2.004539 & 0.337226\end{array}$

$\begin{array}{llll}\text { F } & 0.678301 & -2.788143 & 0.098440\end{array}$

$\begin{array}{llll}\text { F } & 2.329730 & -1.755401 & -0.879112\end{array}$

$\begin{array}{llll}\text { F } & 2.647010 & -2.782821 & 1.006868\end{array}$

$\begin{array}{llll}\mathrm{O} & 0.474968 & -0.040539 & 0.500667\end{array}$

$\begin{array}{llll}\mathrm{H} & -0.740089 & 0.853594 & -1.399663\end{array}$

\section{$\mathrm{CH}_{3} \mathrm{CHOHCF}_{2}^{-}$}

$\begin{array}{lrrr}\mathrm{C} & 0.000000 & 0.000000 & 0.000000 \\ \mathrm{C} & 0.000000 & 0.000000 & 1.521525 \\ \mathrm{C} & 1.421502 & 0.000000 & 2.095127 \\ \mathrm{O} & -0.847701 & 1.100985 & 1.957919 \\ \mathrm{H} & 0.570001 & -0.855368 & -0.373391 \\ \mathrm{C} & -1.024140 & -0.054355 & -0.385262 \\ \mathrm{H} & 0.466392 & 0.917494 & -0.367528 \\ \mathrm{H} & -0.454510 & -0.933922 & 1.886298 \\ \mathrm{H} & -0.466285 & 1.354603 & 2.809002 \\ \mathrm{~F} & 1.967980 & 1.297205 & 1.698673 \\ \mathrm{~F} & 1.142585 & 0.290478 & 3.536484\end{array}$

\section{$\mathrm{CF}_{3} \mathrm{CO}_{2} \mathrm{H}$}

$\begin{array}{llll}\mathrm{C} & 0.000000 & 0.000000 & 0.000000 \\ \mathrm{C} & 0.000000 & 0.000000 & 1.552547 \\ \mathrm{O} & 0.996202 & 0.000000 & 2.212099\end{array}$




$\begin{array}{lrrr}\text { F } & 1.247485 & 0.000000 & -0.465322 \\ \text { F } & -0.631765 & -1.089394 & -0.471053 \\ \text { F } & -0.631765 & 1.089394 & -0.471053 \\ \text { O } & -1.257229 & 0.000000 & 2.005706 \\ \text { H } & -1.232815 & 0.000000 & 2.975385\end{array}$

\section{$\mathrm{CH}_{3} \mathrm{CHOHCHF}_{2}$}

$\mathrm{C} \quad 0.000000$

C $\quad 0.000000$

$\begin{array}{ll}0.000000 & 0.000000\end{array}$

C $\quad 1.414221$

$0.000000 \quad 1.520754$

O $\quad-0.730226$

$0.000000 \quad 2.092144$

$1.132509 \quad 1.961053$

$\mathrm{H} \quad 0.545137$

$-0.862767$

$-0.392140$

H $\quad-1.027826$

$-0.047435$

$-0.362006$

$\mathrm{H} \quad 0.462095$

0.913406

$-0.377454$

$-0.925214 \quad 1.892172$

$\mathrm{H} \quad-0.466921$

1.182608

2.922686

H $\quad-0.680071$

1.075318

1.663655

F $\quad 1.339798$

0.094576

3.469938

H 1.976139

$-0.906831$

1.854984

\section{$\mathrm{CF}_{3} \mathrm{CO}_{2}^{-}$}

$\begin{array}{lrrr}\mathrm{C} & 0.000000 & 0.000000 & 0.000000 \\ \mathrm{C} & 0.000000 & 0.000000 & 1.591552 \\ \mathrm{O} & 1.126772 & 0.000000 & 2.109142 \\ \mathrm{~F} & 1.232574 & 0.000000 & -0.573572 \\ \mathrm{~F} & -0.642473 & -1.089870 & -0.518490 \\ \mathrm{~F} & -0.642473 & 1.089870 & -0.518490 \\ \mathrm{O} & -1.149560 & 0.000000 & 2.060233\end{array}$

Table S2. Total energy ( $E$, hartree), Zero-point Vibrational Energy (ZPVE, kcal/mol), Thermal Correction to Enthalpy $\left(\Delta H_{\mathrm{c}}, \mathrm{kcal} / \mathrm{mol}\right)$, and Absolute Entropy $(S$, eu) Calculated at the B3LYP/6-311+G(d,p) Level of Theory for Various Species Involved in the Hydroxide Ion-Initiated C-C Bond Cleavages of 1,1,1,3,3-Pentafluoro-4-hydroxypentan-2-one (R) in the Gas Phase

\begin{tabular}{lcccc}
\hline species & $E$ & $Z P V E$ & $\Delta H_{\mathrm{c}}(298 \mathrm{~K})$ & $S(298 \mathrm{~K})$ \\
\hline R & -843.445636 & 66.8 & 74.9 & 109.6 \\
HO $^{-}$ & -75.827331 & 5.4 & 7.4 & 41.2 \\
INT & -919.392378 & 75.4 & 84.0 & 111.1 \\
TS1 & -919.354309 & 73.6 & 82.7 & 117.0 \\
CX1 & -919.365937 & 72.4 & 82.4 & 131.9 \\
TS2 & -919.362564 & 73.2 & 82.6 & 125.6 \\
CX2 & -919.439466 & 75.4 & 84.9 & 128.8 \\
TS3 & -919.339521 & 73.1 & 82.3 & 118.5 \\
CX3 & -919.342410 & 72.7 & 82.6 & 131.1 \\
TS4 & -919.340201 & 72.7 & 82.2 & 126.1 \\
CX4 & -919.443454 & 75.4 & 85.0 & 133.5
\end{tabular}




\begin{tabular}{lcccc}
$\mathrm{CH}_{3} \mathrm{CHOHCF}_{2} \mathrm{CO}_{2} \mathrm{H}$ & -581.587624 & 67.5 & 73.9 & 94.4 \\
$\mathrm{CF}_{3}^{-}$ & -337.740179 & 5.5 & 8.5 & 68.1 \\
$\mathrm{CH}_{3} \mathrm{CHOHCF}_{2} \mathrm{CO}_{2}^{-}$ & -581.069506 & 59.2 & 65.3 & 91.8 \\
$\mathrm{CHF}_{3}$ & -338.349138 & 15.7 & 18.4 & 62.1 \\
$\mathrm{CH}_{3} \mathrm{CHOHCF}_{2}^{-}$ & -392.340554 & 48.5 & 53.4 & 80.0 \\
$\mathrm{CF}_{3} \mathrm{CO}_{2} \mathrm{H}$ & -526.965524 & 24.3 & 28.8 & 81.3 \\
$\mathrm{CH}_{3} \mathrm{CHOHCHF}_{2}$ & -392.966610 & 58.5 & 63.2 & 79.7 \\
$\mathrm{CF}_{3} \mathrm{CO}_{2}^{-}$ & -526.444753 & 15.9 & 20.3 & 82.4 \\
\hline
\end{tabular}

Table S3. Total energy (hartree) in Solution Calculated at the B3LYP/6-311+G(d,p) Level with the PCM Method for Various Species Involved in the Hydroxide Ion-Initiated $\mathrm{C}-\mathrm{C}$ Bond Cleavages of 1,1,1,3,3-Pentafluoro-4-hydroxypentan-2-one $(\mathbf{R})^{\mathrm{a}}$

\begin{tabular}{lcc}
\hline species & THF & water \\
\hline $\mathbf{R}$ & -843.449513 & -843.456967 \\
$\mathrm{HO}^{-}$ & -75.954121 & -75.995284 \\
$\mathbf{I N T}$ & -919.459513 & -919.483675 \\
$\mathbf{T S 1}$ & -919.416963 & -919.440907 \\
$\mathbf{C X 1}$ & -919.429568 & nc \\
$\mathbf{T S 2}$ & -919.429407 & -919.452843 \\
$\mathbf{C X 2}$ & -919.503720 & -919.520579 \\
$\mathbf{T S 3}$ & -919.399408 & -919.419440 \\
$\mathbf{C X 3}$ & -919.401615 & -919.421422 \\
$\mathbf{T S 4}$ & -919.399712 & -919.421895 \\
$\mathbf{C X 4}$ & -919.504952 & -919.500231 \\
$\mathrm{CH}_{3} \mathrm{CHOHCF}_{2} \mathrm{CO}_{2} \mathrm{H}$ & -581.595448 & -581.608061 \\
$\mathrm{CF}_{3}^{-}$ & -337.825012 & -337.847139 \\
$\mathrm{CH}_{3} \mathrm{CHOHCF}_{2} \mathrm{CO}_{2}{ }^{-}$ & -581.144248 & -581.165245 \\
$\mathrm{CHF}_{3}$ & -338.350927 & -338.352641 \\
$\mathrm{CH}_{3} \mathrm{CHOHCF}_{2}^{-}$ & -392.417721 & -392.444002 \\
$\mathrm{CF}_{3} \mathrm{CO}_{2} \mathrm{H}_{\mathrm{CH}_{3} \mathrm{CHOHCHF}_{2}}^{-}$ & -526.971381 & -526.980342 \\
$\mathrm{CF}_{3} \mathrm{CO}_{2}{ }^{-}$ & -392.971402 & -392.978558 \\
& -526.523025 & -526.544630 \\
\hline
\end{tabular}

${ }^{\mathrm{a}}$ At the geometries optimized in the gas phase at the B3LYP/6$311+\mathrm{G}(\mathrm{d}, \mathrm{p})$ level. 